\title{
Relations entre la qualité du liège, sa composition minérale et la composition minérale des feuilles du chêne-liège (Quercus suber L.)
}

\author{
Michèle Courtois*, Philippe Masson \\ Laboratoire d'ingénierie agronomique, Ensat, avenue de l'Agrobiopôle BP 107, 31326 Castanet-Tolosan cedex, France
}

(Reçu le 4 juin 1998 ; accepté 28 octobre 1998)

\begin{abstract}
Relationships between the quality of cork, its inorganic content and the inorganic content of the leaves of the corkoak (Quercus suber L.). The aim of this work is to set up a relationship between the quality of cork, its inorganic content and the inorganic content of the leaves of the cork-oak. The mineral contents of both leaves and cork were identified for a range of elements (nitrogen, phosphorus, potassium, calcium, magnesium, iron, copper, zinc and manganese). The quality of the cork was evaluated by experts in the cork industry on the basis of visual assessment of cork stoppers resulting from each tree. A study of correlations between the cork inorganic contents and the quality of cork demonstrates that, among those measures, only the potassium content of cork is related to the cork quality. The cork quality seems to be independent of the leaf inorganic content, which is held to be an indicator of the cork-oak mineral nutrition. (ㅇ Inra/Elsevier, Paris.)
\end{abstract}

Quercus suber L / cork / leaf / inorganic content / quality

Résumé - Le but de ce travail est de déterminer parmi des analyses minérales de feuilles et de liège, un ou plusieurs éléments minéraux dont la teneur pourrait être en relation avec la qualité du liège. La composition minérale des feuilles et du liège a donc été déterminée pour un certain nombre d'éléments (azote, pḥosphore, potassium, calcium, magnésium, fer, cuivre, zinc et manganèse). La qualité du liège est estimée par des experts de l'industrie du liège à partir des ratios de choix visuels des bouchons obtenus de chaque arbre. L'étude des corrélations entre les teneurs en éléments minéraux du liège et sa qualité indique que, parmi ces mesures, seule la teneur en potassium du liège est liée à la qualité du matériau. De plus, la qualité du liège semble être indépendante de la composition minérale des feuilles, considérée comme un indicateur de la nutrition minérale du chêne-liège. (@ Inra/Elsevier, Paris.)

Quercus suber L / liège / feuille / composition minérale / qualité

\section{Introduction}

La demande de liège de la part des industries de la bouchonnerie se porte de plus en plus sur des lièges susceptibles de donner des bouchons de haute qualité pour une parfaite conservation des vins, notamment des vins d'appellation. Les industriels du liège achètent chaque année aux propriétaires de chênes-lièges, le droit de récolter directement le liège sur l'arbre. Les entreprises réalisent donc leurs achats directement en forêt en procédant, un à deux mois avant la levée, à des prélèvements d'échantillons de liège sur des chêneslièges représentatifs de la forêt. Les conditions financières dépendent de la quantité mais surtout de la quali-

* Correspondance et tirés à part apwagner@t-online.de 
té du liège levé. La connaissance exacte de la qualité du liège avant la levée est donc essentielle dans la détermination correcte des niveaux de paiement pour le matériau brut. Mais le liège est un matériau naturel présentant une hétérogénéité qui rend difficile toute normalisation. Actuellement, la classification de la qualité est faite en utilisant principalement des paramètres subjectifs qui d'un point de vue technique ne sont pas entièrement satisfaisants. En effet, les lièges sont classés essentiellement d'après des qualités de texture appréciées à l'œil et au toucher. L'appréciation de cette qualité visuelle repose uniquement sur l'expérience professionnelle de l'opérateur. Cette méthode de classification demande donc une longue expérience et reste assez subjective.

Le but de cette étude est de trouver des paramètres de qualité du liège plus facilement mesurables comme des analyses minérales qui permettraient de mieux quantifier la qualité du matériau. De nombreux éléments minéraux ont été identifiés par Barcelo [1] : $\mathrm{Ca}, \mathrm{K}, \mathrm{Mn}, \mathrm{Fe}, \mathrm{Al}$, $\mathrm{Mg}, \mathrm{Na}, \mathrm{Ba}, \mathrm{Sr}, \mathrm{Cu}, \mathrm{Li}, \mathrm{Cr}$ et $\mathrm{Ti}$. Le rôle de ces éléments dans le matériau liège n'est pas encore connu. C'est pourquoi, il est intéressant de réaliser des analyses minérales du liège pour étudier les relations entre sa composition minérale et sa qualité.

De plus, pour vérifier l'hypothèse d'une liaison entre composition minérale du liège et nutrition minérale, des analyses minérales de feuilles des chênes-lièges sont nécessaires. De nombreux auteurs ont montré que la nutrition minérale du chêne-liège joue un rôle important sur la croissance de celui-ci ainsi que sur la production quantitative de liège. En 1981, Zeraia [14] arrive à la conclusion que l'azote favorise la croissance du liège. Les traitements sylvopastoraux ont aussi un effet positif significatif sur la croissance du chêne-liège : croissance apicale et surface foliaire [9]. L'amélioration de cette croissance est attribuée à l'augmentation de la disponibilité de l'eau et des éléments limitants $N$ et $P$ [12]. Orgeas [11] montre que la meilleure production quantitative de liège est liée à une plus grande teneur en $\mathrm{K}$ et $\mathrm{N}$ des feuilles. Donc, pour savoir si la nutrition de l'arbre a également une répercussion sur la production en qualité du liège, des analyses minérales de feuilles ont été réalisées et des études de corrélations entre teneurs en certains éléments et qualité du matériau brut ont été menées.

Le travail présenté dans cet article fait partie d'une étude plus vaste comprenant également l'évaluation de la qualité du liège par des paramètres physiques du liège. Des liaisons significatives entre qualité visuelle du liège estimée par les experts et masse volumique du liège ainsi que force de compression du liège ont été trouvées et seront données ultérieurement.

\section{Matériel et méthodes}

\subsection{Sites}

Quatre sites représentatifs des zones de production de chênes-lièges sont sélectionnés : la forêt $\mathrm{A}$ en Andalousie (Espagne, province de Cadix, localité Medina-Sidonia), la forêt B en Estrémadure (Espagne, province de Badajoz, localité Zahinos), la forêt $\mathrm{C}$ en Estrémadure (Espagne, province de Caceres, localité Aliseda) et la forêt D en Catalogne (France, arrondissement de Céret, localité Maureillas). Ces forêts se situent dans des régions à climat méditerranéen : subhumide de tendance atlantique pour la forêt $\mathrm{A}$, semi-aride et chaud pour les forêts $B$ et $C$, subhumide pour la forêt $D$. Ces sites se trouvent sur sols acides. La nature de la roche mère est siliceuse pour toutes les forêts. Les sites B et C sont des suberaies de type « dehesa », composée de chênes-lièges en peuplements clairs permettant la présence d'un sous-étage herbacé diversifié (graminées, légumineuses) ainsi que le pâturage de troupeaux de moutons ou de chèvres. Le site $\mathrm{A}$ mais surtout le site $\mathrm{D}$ sont plutôt de type «forêt » avec une forte densité d'arbres à l'hectare et avec un sous-étage arbustif dense de type maquis.

\subsection{Méthodes d'échantillonnage}

Un premier échantillonnage a été fait sur un grand nombre de chênes-lièges (100 arbres par propriété) suivant une ligne représentative de la topographie et du peuplement des propriétés. Diverses caractéristiques des arbres sont mesurées : diamètre et hauteur de l'arbre, diamètre de canopée, hauteur de déliègeage, épaisseur du liège et volume de liège démasclé. La qualité visuelle du liège est estimée sur des morceaux de liège de $10 \mathrm{~cm}$ sur $10 \mathrm{~cm}$ prélevés sur la face Est de chaque tronc d'arbre, toujours à la même hauteur $(1,30 \mathrm{~m})$.

Les coefficients de corrélation (r) entre la qualité du liège et les variables mesurées sur les arbres ont été calculés. Il ressort que seule l'épaisseur du liège a une relation significative avec la qualité visuelle de celui-ci ( $r=0,64$ pour l'ensemble des forêts). L'objectif étant de faire un échantillonnage de la qualité intrinsèque du liège plutôt qu'un échantillonnage d'épaisseur du liège, le facteur « épaisseur »a été éliminé en choisissant un souséchantillon de 30 arbres par propriété dans les deux principaux calibres de liège bouchonnable : la classe 13/15 et la classe 15/20 (en quantité égale). C'est sur ce souséchantillon que sont faits les prélèvements de feuilles et de liège pour les analyses chimiques. Ces prélèvements ont lieu de juin à juillet, dans la période de levée des chênes-lièges. Les arbres sont entièrement déliègés et les 
planches de liège sont transformées en bouchons. Des rameaux de feuilles matures sont prélevés au niveau de la partie médiane de la couronne de chaque arbre, dans quatre directions opposées. Les feuilles âgées sont jugées les plus favorables à un bon diagnostic foliaire du chêneliège [12].

\subsection{Méthodes d'analyses minérales}

Les analyses minérales sont réalisées à partir de poudre résultant d'une part du broyage des feuilles séchées et d'autre part du ponçage de morceaux de liège [12]. Les teneurs des macroéléments ( $\mathrm{N}, \mathrm{P}, \mathrm{K}, \mathrm{Ca}, \mathrm{Mg}$ ) et oligoéléments $(\mathrm{Fe}, \mathrm{Cu}, \mathrm{Zn}, \mathrm{Mn})$ sont déterminées par des méthodes classiques [12]. L'azote est minéralisé dans l'acide sulfurique $\mathrm{H}_{2} \mathrm{SO}_{4}$ en présence d' $\mathrm{H}_{2} \mathrm{O}_{2}$ selon la méthode de Lindner [5]. Les autres éléments sont minéralisés par calcination de $2 \mathrm{~g}$ de matière sèche à $550{ }^{\circ} \mathrm{C}$ selon la méthode de Bonvalet [2]. Les cendres sont dissoutes avec $\mathrm{HCl}$ (volume final $50 \mathrm{~mL} \mathrm{HCl} 0,5 \mathrm{~N}$ ). L'azote et le phosphore sont dosés par spectrophotocolorimètrie, en utilisant la méthode au bleu d'indophénol $(625 \mathrm{~nm})$ et le complexe phosphomolybdique $(660 \mathrm{~nm})$ respectivement. Les autres macroéléments $(\mathrm{Ca}, \mathrm{Mg})$ et les oligoéléments sont dosés par spectrophotomètrie d'absorption atomique ou d'émission atomique $(K)$.

\subsection{Méthode industrielle d'estimation de la qualité du liège}

Les planches de liège échantillonnées sont laissées un an à l'air libre. Après bouillage et passage en cave de maturation, elles sont transformées en bouchons. L'évaluation de la qualité du liège d'un arbre se fait à partir des ratios de choix visuels des bouchons. Les choix sont déterminés sur 200 bouchons par arbre, pris au hasard. Les choix des bouchons sont classés par ordre de qualité visuelle décroissante : super, $1,2,3,4,5,6$ et déchets. Les choix super, 1, 2, 3, 4, 5 et 6 sont fonction du nombre et de la taille des lenticelles ainsi que des éventuels défauts mineurs présents sur le bouchon. Le choix de chaque bouchon est déterminé par des experts de l'industrie du liège.

La répartition des choix faite sur les bouchons provenant de chaque arbre étudié permet de donner la qualité du liège de l'arbre entier. Chaque choix a une valeur basée sur le prix de vente des bouchons (tableau I). Un indice de qualité est calculé pour chaque arbre à partir des pourcentages de tous les choix de bouchons et de leur valeur (moyenne pondérée). Les indices de qualité du liège obtenus peuvent se diviser en trois groupes de
Tableau I. Échelle de valeur des choix des bouchons (sans unité).

\begin{tabular}{lcccccccc}
\hline Choix & Super & 1 & 2 & 3 & 4 & 5 & 6 & Déchet \\
Valeur & 182 & 161 & 114 & 100 & 71 & 48 & 39 & 20 \\
\hline
\end{tabular}

qualité visuelle : inférieur à 48 (basse qualité), de 48 à 114 (bonne qualité), de 114 à 182 (haute qualité).

\subsection{Méthode d'analyse statistique}

Les corrélations entre qualité visuelle du liège et éléments minéraux des feuilles et du liège sont étudiées à l'aide du coefficient de corrélation linéaire de BravaisPearson (coefficient r). Pour l'étude de l'ensemble des quatre forêts (120 arbres échantillonnés), le coefficient de corrélation est significativement différent de zéro si $|r|>0,18$ au seuil de sécurité de $95 \%$. Pour l'étude portant sur une forêt ( 30 arbres échantillonnés), le coefficient de corrélation est significativement différent de zéro si $|\mathrm{r}|>0,36$ au seuil de sécurité de $95 \%$.

\section{Résultats}

\subsection{Corrélations entre qualité visuelle du liège et éléments minéraux du liège}

Les résultats concernant les analyses minérales du liège, moyennes des 30 arbres par forêt, sont présentés tableau II. L'ordre de grandeur des teneurs en éléments minéraux du liège est donné : $\mathrm{P}: 0,03$ à $0,04 \% \mathrm{~m} . \mathrm{s}, \mathrm{K}$ : 0,14 à $0,27 \%$ m.s, Ca : 0,11 à $0,20 \%$ m.s, $\mathrm{Mg}: 0,01$ à $0,02 \% \mathrm{~m} . \mathrm{s}, \mathrm{N}: 0,47$ à $0,61 \% \mathrm{~m} . \mathrm{s}, \mathrm{Mn}: 25$ à $52 \mathrm{mg} / \mathrm{kg}$ m.s, $\mathrm{Cu}: 10,6$ à $16,4 \mathrm{mg} \mathrm{kg}^{-1} \mathrm{~m} . \mathrm{s}, \mathrm{Zn}: 7,2$ à $11,0 \mathrm{mg}$ $\mathrm{kg}^{-1} \mathrm{~m}$.s et Fe : 132 à $191 \mathrm{mg} \mathrm{kg}^{-1}$ m.s. L'écart-type des mesures reflète en général les différences entre les arbres étudiés : dans le cas de $\mathrm{Cu}$ et $\mathrm{Zn}$, la variabilité observée est en partie expliquée par la moindre précision du processus analytique pour ces éléments présents à de faibles teneurs dans le liège. À l'heure actuelle, peu de recherches ont été faites concernant les teneurs en éléments minéraux du liège. Leonardi [4] donne les valeurs suivantes pour les macroéléments du liège : $\mathrm{P}: 0,04 \%$ m.s, K : 0,14 \% m.s, Ca : 2,45\% m.s, Mg : 0,01\% m.s, $\mathrm{N}: 0,36 \% \mathrm{~m} . \mathrm{s}$. Les teneurs trouvées dans cette étude sont donc semblables à celles de Leonardi à l'exception de la teneur en $\mathrm{Ca}$ qui est beaucoup plus faible $(0,18 \%$ m.s en moyenne comparée à $2,45 \%$ m.s pour Leonardi). Cette différence pourrait venir d'une différence de teneurs en $\mathrm{Ca}$ du sol ou d'une différence de disponibilité de Ca. 
Tableau II. Composition minérale du liège (moyennes de 30 échantillons par forêt \pm déviation standard).

\begin{tabular}{|c|c|c|c|c|c|c|c|c|c|}
\hline Liège & \multicolumn{5}{|c|}{ en \% matière sèche } & \multicolumn{4}{|c|}{ en $\mathrm{mg} \mathrm{kg}^{-1}$ matière sèche } \\
\hline $\begin{array}{l}\text { Forêt B } \\
(n=30)\end{array}$ & $\begin{array}{c}0,040 \\
\pm 0,014\end{array}$ & $\begin{array}{c}0,237 \\
\pm 0,09 I\end{array}$ & $\begin{array}{c}0,132 \\
\pm 0,046\end{array}$ & $\begin{array}{c}0,017 \\
\pm 0,008\end{array}$ & $\begin{array}{c}0,615 \\
\pm 0,057\end{array}$ & $\begin{array}{c}35 \\
\pm 11,4\end{array}$ & $\begin{array}{c}16,4 \\
\pm 5,0\end{array}$ & $\begin{array}{c}10,9 \\
\pm 5,7\end{array}$ & $\begin{array}{c}157 \\
\pm 91\end{array}$ \\
\hline $\begin{array}{l}\text { Forêt C } \\
(n=30)\end{array}$ & $\begin{array}{c}0,035 \\
\pm 0,014\end{array}$ & $\begin{array}{c}0,270 \\
\pm 0,076\end{array}$ & $\begin{array}{c}0,113 \\
\pm 0,043\end{array}$ & $\begin{array}{c}0,011 \\
\pm 0,004\end{array}$ & $\begin{array}{c}0,472 \\
\pm 0,049\end{array}$ & $\begin{array}{c}25 \\
\pm 8,7\end{array}$ & $\begin{array}{l}13,0 \\
\pm 2,7\end{array}$ & $\begin{array}{c}7,7 \\
\pm 3,9\end{array}$ & $\begin{array}{l}132 \\
\pm 68\end{array}$ \\
\hline $\begin{array}{l}\text { Forêt D } \\
(n=30)\end{array}$ & $\begin{array}{c}0,027 \\
\pm 0,011\end{array}$ & $\begin{array}{c}0,136 \\
\pm 0,066\end{array}$ & $\begin{array}{c}0,198 \\
\pm 0,074\end{array}$ & $\begin{array}{c}0,013 \\
\pm 0,006\end{array}$ & $\begin{array}{c}0,562 \\
\pm 0,067\end{array}$ & $\begin{array}{c}52 \\
\pm 22,7\end{array}$ & $\begin{array}{r}10,6 \\
\pm 3,5\end{array}$ & $\begin{array}{c}11,0 \\
\pm 6,2\end{array}$ & $\begin{array}{r}191 \\
\pm 81\end{array}$ \\
\hline
\end{tabular}

Tableau III. Coefficients de corrélation $r$ entre les indices de qualité du liège et les teneurs en éléments minéraux du liège.

\begin{tabular}{lcccccccccc}
\hline Liège & $\mathrm{P}$ & $\mathrm{K}$ & $\mathrm{Ca}$ & $\mathrm{Mg}$ & $\mathrm{N}$ & $\mathrm{Mn}$ & $\mathrm{Cu}$ & $\mathrm{Zn}$ & $\mathrm{Fe}$ \\
\hline Forêt $\mathrm{A}(n=30)$ & $-0,24$ & 0,10 & $-0,08$ & 0,15 & $-0,15$ & $-0,03$ & $-0,17$ & 0,19 & 0,13 \\
Forêt B $(n=30)$ & $-0,18$ & $\underline{0,36}$ & 0,35 & $-0,23$ & 0,03 & $-0,11$ & $-0,30$ & $-0,10$ & $-0,03$ \\
Forêt C $(n=30)$ & 0,12 & 0,24 & 0,10 & $-0,29$ & 0,03 & $-0,28$ & 0,13 & 0,02 & $-0,13$ \\
Forêt D $(n=30)$ & 0,14 & 0,32 & $-0,14$ & $-0,08$ & 0,04 & 0,13 & $-0,27$ & $-0,17$ & $-0,13$ \\
Totalité des forêts $(n=120)$ & 0,05 & $\underline{0,23}$ & $-0,08$ & 0,01 & $-0,06$ & $-0,14$ & $-0,01$ & $-0,07$ & $-0,10$ \\
\hline
\end{tabular}

Les coefficients de corrélation entre indices de qualité du liège et teneurs en $\mathrm{P}, \mathrm{K}, \mathrm{Ca}, \mathrm{Mg}, \mathrm{N}, \mathrm{Mn}, \mathrm{Cu}, \mathrm{Zn}$ et Fe du liège sont présentés dans le tableau III. Une corrélation positive est significative, entre les indices de qualité du liège et la teneur en $\mathrm{K}$ du liège pour la totalité des forêts $(r=0,23)$. La qualité du liège augmente avec la teneur en $\mathrm{K}$ de celui-ci. En revanche, si on considère les forêts prises individuellement, les coefficients de corrélation ne sont pas tous significatifs. Cela signifie qu'il y a une influence des caractéristiques du site. La corrélation entre qualité du liège et teneur en $\mathrm{K}$ du liège est plus significative dans la forêt $\mathrm{B}$ que dans les autres sites. En effet, cette forêt a été semée de trèfle souterrain et fertilisée par $\mathrm{P}_{2} \mathrm{O}_{5}$. Cela a pour effet d'améliorer la teneur en $\mathrm{P}$ et $\mathrm{N}$ du sol et par conséquent les teneurs des feuilles du chêne-liège ainsi que du liège. $\mathrm{N}$ et $\mathrm{P}$ sont donc non limitants, ce qui a pour conséquence d'améliorer la corrélation entre qualité du liège et teneur en K. Quand on prend en compte la globalité des forêts, on efface l'effet site et on obtient une relation qui est intrinsèque au matériau liège. Cependant, le coefficient de corrélation entre la teneur en $\mathrm{K}$ du liège et la qualité visuelle de celui-ci est faible. La figure I présente le diagramme de dispersion, pour la totalité des forêts, entre la teneur en $\mathrm{K}$ du

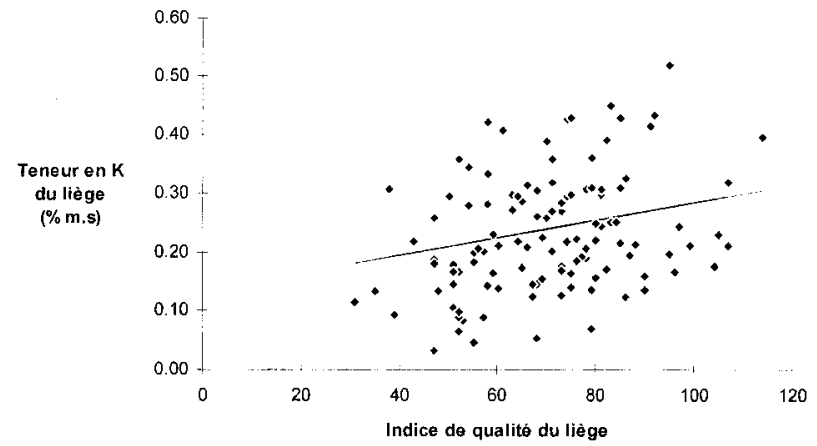

Figure 1. Diagramme de dispersion entre teneur en $\mathrm{K}$ du liège et indice de qualité du liège pour l'ensemble des forêts $(n=120)$.

liège et les indices de qualité du liège. Ce diagramme montre la grande relativité de cette liaison. En effet, il y a une forte variabilité autour de la droite de régression et donc aussi à l'intérieur des trois groupes de qualité visuelle du liège. Si on considère les données obtenues, pour une basse qualité de liège (indices de qualité inférieurs à 48), la moyenne des teneurs en $\mathrm{K}$ est de $0,20 \%$ 
Tableau IV. Composition minérale des feuilles de chêne-liège (moyennes de 30 échantillons par forêt \pm déviation standard).

\begin{tabular}{|c|c|c|c|c|c|c|c|c|c|}
\hline Feuilles & \multicolumn{5}{|c|}{ en $\%$ matière sèche } & \multicolumn{4}{|c|}{ en $\mathrm{mg} \mathrm{kg}^{-1}$ matière sèche } \\
\hline $\begin{array}{l}\text { Forêt B } \\
(n=30)\end{array}$ & $\begin{array}{c}0,092 \\
\pm 0,033\end{array}$ & $\begin{array}{c}0,440 \\
\pm 0,073\end{array}$ & $\begin{array}{c}0,648 \\
\pm 0,149\end{array}$ & $\begin{array}{c}0,211 \\
\pm 0,042\end{array}$ & $\begin{array}{c}1,57 \\
\pm 0,20\end{array}$ & $\begin{array}{c}825 \\
\pm 262\end{array}$ & $\begin{array}{c}3,74 \\
\pm I, 5 I\end{array}$ & $\begin{array}{l}16,2 \\
\pm 3,0\end{array}$ & $\begin{array}{c}164 \\
\pm 28\end{array}$ \\
\hline $\begin{array}{l}\text { Forêt C } \\
(n=30)\end{array}$ & $\begin{array}{c}0,069 \\
\pm 0,013\end{array}$ & $\begin{array}{c}0,512 \\
\pm 0,131\end{array}$ & $\begin{array}{c}0,750 \\
\pm 0,180\end{array}$ & $\begin{array}{c}0,152 \\
\pm 0,031\end{array}$ & $\begin{array}{c}1,13 \\
\pm 0,18\end{array}$ & $\begin{array}{c}994 \\
\pm 439\end{array}$ & $\begin{array}{c}3,91 \\
\pm 0,72\end{array}$ & $\begin{array}{l}13,5 \\
\pm 2,4\end{array}$ & $\begin{array}{l}162 \\
\pm 29\end{array}$ \\
\hline $\begin{array}{l}\text { Forêt D } \\
(n=30)\end{array}$ & $\begin{array}{c}0,079 \\
\pm 0,011\end{array}$ & $\begin{array}{c}0,584 \\
\pm 0,150\end{array}$ & $\begin{array}{c}0,678 \\
\pm 0,115\end{array}$ & $\begin{array}{c}0,190 \\
\pm 0,049\end{array}$ & $\begin{array}{c}1,24 \\
\pm 0,17\end{array}$ & $\begin{array}{c}1838 \\
\pm 586\end{array}$ & $\begin{array}{c}4,15 \\
\pm 0,81\end{array}$ & $\begin{array}{c}17,0 \\
\pm 2,7\end{array}$ & $\begin{array}{r}186 \\
\pm 34\end{array}$ \\
\hline
\end{tabular}

m.s avec un intervalle de confiance de $(0,10,0,29)$. Pour une bonne qualité de liège (indices de qualité compris entre 48 et 114), la moyenne des teneurs en $\mathrm{K}$ est de $0,24 \% \mathrm{~m} . \mathrm{s}$ avec un intervalle de confiance de $(0,22$, $0,26)$. L'amplitude des variations dans chaque catégorie et les recoupements des intervalles sont trop importants pour pouvoir retenir une échelle de qualité du liège.

On remarque un autre coefficient de corrélation proche du seuil de signification, $(r=0,35)$, entre la qualité du liège et sa teneur en $\mathrm{Ca}$, pour la forêt $\mathrm{B}$. Seulement, cette liaison positive ne se retrouve pas au niveau des autres forêts. Elle n'est donc pas caractéristique du matériau liège.

Aucune relation significative entre la qualité visuelle du liège et les teneurs en autres macroéléments du liège, à savoir, P, Mg, N, et les oligoéléments n'a pu être établie.

\subsection{Corrélations entre qualité visuelle du liège et éléments minéraux des feuilles}

Les teneurs en éléments minéraux des feuilles de chêne-liège (tableau IV) appartiennent aux fourchettes de valeurs rencontrées dans la littérature existante sur la nutrition minérale du chêne-liège $[10,12]: \mathrm{P}(0,08$ à $0,20 \%$ m.s $), \mathrm{K}(0,35$ à $1,03 \%$ m.s $), \mathrm{Ca}(0,23$ à $1,06 \%$ m.s), Mg (0,09 à 0,26\% m.s), N (1,05 à $1,88 \%$ m.s $)$, Mn (855 à $\left.1699 \mathrm{mg} \mathrm{kg}^{-1} \mathrm{~m} . \mathrm{s}\right), \mathrm{Cu}\left(4,48\right.$ à $6,29 \mathrm{mg} \mathrm{kg}^{-1}$ m.s), Zn (16,6 à 26,0 mg kg-1 m.s) et Fe (154 à $251 \mathrm{mg}$ $\left.\mathrm{kg}^{-1} \mathrm{~m} . \mathrm{s}\right)$.

La qualité visuelle du liège estimée sur bouchons est mise en relation avec les teneurs en $\mathrm{P}, \mathrm{K}, \mathrm{Ca}, \mathrm{Mg}, \mathrm{N}$, $\mathrm{Mn}, \mathrm{Cu}, \mathrm{Zn}$ et Fe contenues dans les feuilles (tableau V).
Un seul coefficient de corrélation est significatif, $(r=-0,37)$, entre la qualité du liège estimée à partir des bouchons et la teneur en fer des feuilles de la forêt $\mathrm{A}$. L'indice de qualité du liège diminue quand augmente la teneur en fer des feuilles. Seulement, ce critère n'a pas été validé pour les autres forêts où les coefficients de corrélation sont non significatifs et où la tendance est quelquefois inverse ( $r$ positif). L'influence de la teneur en fer des feuilles sur la qualité du liège n'est pas propre au matériau liège mais est dépendante du site soit en raison des facteurs du milieu soit des facteurs génétiques. Cette mesure chimique ne peut donc pas être retenue comme critère de qualité du liège.

On remarque aussi une faible liaison entre teneur en $\mathrm{N}$ des feuilles et qualité du liège. Les coefficients ne sont pas significatifs mais ils vont tous dans le même sens : effet positif surtout pour les forêts B et C $(r=0,28$ / $0,26)$. Cela ne représente qu'une tendance qui ne peut être prise pour l'évaluation de la qualité du liège.

D'autre part, les résultats n'ont pas permis d'établir de relation significative entre la qualité visuelle du liège et les autres éléments minéraux analysés dans les feuilles. D'après cette étude, la qualité visuelle du liège semble indépendante de la composition minérale des feuilles des chênes-lièges et de la nutrition minérale de l'arbre.

\section{Discussion}

D'après les résultats, la composition minérale du liège est peu corrélée à la teneur en éléments minéraux des feuilles. Pour l'ensemble des forêts, seules les liaisons entre teneurs des feuilles et teneurs du liège en $\mathrm{N}$ ainsi qu'en $\mathrm{Mn}$ se remarquent par leur coefficient de corrélation significatif (respectivement 0,38 et 0,44 ). Ce 
Tableau V. Coefficients de corrélation $r$ entre les indices de qualité du liège et les teneurs en éléments minéraux des feuilles.

\begin{tabular}{lcccccccccc}
\hline Feuilles & $\mathrm{P}$ & $\mathrm{K}$ & $\mathrm{Ca}$ & $\mathrm{Mg}$ & $\mathrm{N}$ & $\mathrm{Mn}$ & $\mathrm{Cu}$ & $\mathrm{Zn}$ & $\mathrm{Fe}$ \\
\hline Forêt A $(n=30)$ & 0,25 & $-0,12$ & $-0,16$ & 0,02 & 0,09 & $-0,28$ & 0,21 & $-0,08$ & $-0,37$ \\
Forêt B $(n=30)$ & 0,16 & 0,11 & $-0,13$ & 0,19 & 0,28 & 0,10 & $-0,22$ & $-0,10$ & $-0,18$ \\
Forêt C $(n=30)$ & $-0,28$ & 0,06 & 0,29 & 0,00 & 0,26 & $-0,12$ & $-0,01$ & $-0,29$ & 0,16 \\
Forêt D $(n=30)$ & $-0,04$ & 0,11 & $-0,19$ & 0,12 & 0,06 & 0,11 & 0,20 & 0,06 & 0,05 \\
Totalité des forêts $(n=120)$ & $-0,06$ & $-0,06$ & 0,04 & 0,06 & 0,07 & $-0,15$ & $-0,05$ & $-0,16$ & $-0,18$ \\
\hline
\end{tabular}

manque de corrélation peut s'expliquer par le fait que la teneur des feuilles est représentative de la nutrition minérale correspondant à l'année de prélèvement tandis que la composition minérale du liège concerne les dix à douze années précédantes. Ainsi, la teneur en $\mathrm{K}$ des feuilles n'est pas liée à la qualité visuelle du liège malgré la liaison teneur en $\mathrm{K}$ du liège et qualité. D'ailleurs, il n'y a pas de liaison entre teneur en $\mathrm{K}$ des feuilles et teneur en $\mathrm{K}$ du liège.

Cette étude montre que $\mathrm{K}$ est le seul élément dont la teneur semble avoir une liaison significative avec la qualité du liège quelle que soit la forêt étudiée. On peut alors se demander de quelle façon la teneur en $\mathrm{K}$ du liège est liée à la qualité visuelle de celui-ci. $\mathrm{K}$ est connu pour être le «maître - cation » de la plante, il est un activateur général du métabolisme. Il se trouve en grande quantité dans les organes qui en ont besoin, là où l'activité physiologique est très intense et où les protéines cellulaires sont en construction. Il joue un rôle positif dans les divisions cellulaires, d'où son importance dans les phénomènes de croissance [8]. D'ailleurs, des études précédentes ont montré que les fortes teneurs en $\mathrm{K}$ favorisent l'accroissement en épaisseur du liège $[11,13]$. De plus, même si la nutrition minérale en $K$ ne semble pas influencer directement la qualité visuelle, elle peut déterminer indirectement la porosité (donc la qualité) à travers son influence sur la croissance. Il se peut aussi que $\mathrm{K}$ améliore la qualité du liège en favorisant le fonctionnement du phellogène. $K$ a également un rôle spécifique dans l'économie de l'eau. K participe à l'augmentation de la pression osmotique et au contrôle des stomates. En activant leur fermeture, il limite la transpiration dès que celle-ci tend à s'exagérer ; à l'inverse, en activant l'ouverture il favorise la photosynthèse lorsque le déficit d'eau n'est pas limitant [7]. En outre, il favorise l'absorption d'eau par les racines [8]. $\mathrm{K}$ pourrait donc améliorer la qualité du liège par son influence sur l'alimentation en eau de l'arbre. Par ailleurs, la disponibilité de $\mathbf{K}$ du sol dépend de la teneur en eau du sol et l'absorption de K est favorisée par une meilleure alimentation hydrique du végétal [7]. Ainsi, de fortes teneurs en $\mathrm{K}$ seraient synonymes de bonne alimentation hydrique et de bonne qualité de liège. A l'inverse, de faibles teneurs en $\mathrm{K}$ seraient liées à une faible alimentation hydrique et à une moins bonne qualité de liège.

Il serait également intéressant de déterminer les paramètres qui influencent la teneur en $\mathrm{K}$ dans le liège et ainsi de connaître les facteurs qui pourraient améliorer la qualité du liège. Ces facteurs qui contribuent à une teneur idéale en $\mathrm{K}$ dans le liège seront néanmoins difficiles à définir. L'hypothèse de contrôler la teneur en $\mathrm{K}$ dans le liège par un apport en $\mathrm{K}$ au sol n'est pas évidente car il a été montré que la teneur en $\mathrm{K}$ du liège n'est pas systématiquement liée à la teneur en $\mathrm{K}$ du sol. D'autre part, les conditions abiotiques locales peuvent varier d'arbre en arbre et influencer la nutrition de l'arbre et la croissance du liège. De plus, la variabilité de la teneur en $\mathrm{K}$ du liège vient sans doute, pour une part, de la forte variabilité génétique des arbres à l'intérieur d'une faible population [6]. En effet, les caractéristiques de reproduction sexuée du chêne-liège, les phénomènes d'hybridation interspécifique et d'allogamie ont permis l'existence et le maintien d'une grande diversité génétique à l'intérieur des populations [3]. Cette haute diversité génétique est conforme à la haute variabilité de la qualité et de la production de liège par les chênes-lièges. La qualité du liège tiendrait, donc, pour une part, au caractère individuel de l'arbre qui le porte.

\section{Conclusion}

L'étude a été menée sur quatre forêts d'Espagne et de France, dans lesquelles des prélèvements de feuilles et de liège ont été fait en vue d'analyses minérales. La qualité visuelle du liège a été estimée par des experts de l'industrie du liège.

L'analyse des corrélations entre qualité visuelle du liège et teneurs en éléments minéraux des feuilles n'a montré aucune liaison simple. D'après les résultats de cette étude, la qualité visuelle du liège n'est pas directement liée à la nutrition minérale de l'arbre. Cela peut provenir de la difficulté de relier la nutrition minérale 
d'une année avec la composition minérale du liège correspondant à une dizaine d'années.

L'étude des corrélations entre la qualité du liège estimée sur bouchons et les teneurs en éléments minéraux du liège des chênes-lièges échantillonnés montre la relation entre qualité et teneur en $\mathrm{K}$ pour l'ensemble des forêts : la qualité du liège augmenterait avec la teneur en $\mathrm{K}$ contenu dans le liège.

Du fait de l'interaction entre alimentation hydrique de l'arbre et sa nutrition en $\mathrm{K}$, la teneur en $\mathrm{K}$ du liège pourrait être influencée par la disponibilité de l'eau dans le sol et par la transpiration de l'arbre. Il paraîtrait donc maintenant nécessaire de mieux préciser les relations entre l'alimentation hydrique et la qualité du liège. Ceci nécessiterait de mieux caractériser l'influence des facteurs de l'environnement des arbres afin de les distinguer des éventuels facteurs génétiques.

Remerciements : Ce travail a été réalisé dans le cadre d'une convention Cifre avec la collaboration des Établissements Sabaté, Bouchons à champagne et à vins fins, 66403 Céret, France. Les auteurs remercient G. Bertoni pour les conseils donnés lors de la relecture de cet article.

\section{Références}

[1] Barcelo, Sobre los elementos metalicos del corcho, Ann. Soc. Esp. Fis. y Quim. 35 (1939) 107.

[2] Bonvalet A., Ferran J., Cassadas E., Analyse minérale de tissus végétaux à faible teneur en silice : Proposition d'une technique de minéralisation simplifiée et d'un plan d'organisation de la méthode d'analyse multiélémentaire, Oléagineux 41 (1986) 141-151.

[3] Garcia-Valdecantos J.L., Elena Rosello J.A., Genetic variability of Quercus suber L. First results of provenance test and isozyme analysis, PROCORK, Workshop 1, European Research on Cork-oak and Cork, Lisbon, 18-19 octobre 1995.
[4] Leonardi S., Rapp M., Failla M., Komaromy E., Biomasse, minéralomasse, productivité et gestion de certains éléments biogènes dans une forêt de Quercus suber $L$. en Sicile (Italie), Ecologia Mediterranea 18 (1992) 89-98.

[5] Lindner R.C., Harley C.P., A rapid method for the determination of nitrogen in plant tissue, Science 96/2503 (1942) $565-566$.

[6] Lumaret, Évaluation de l'état génétique des peuplements de chênes-lièges à l'aide de marqueurs génétiques et relations avec le niveau de régénération (France), Communication au séminaire méditerranéen sur la régénération des forêts de chênes-lièges, Tabarka, 22-23 octobre 1996.

[7] Marschner, Mineral Nutrition of Higher Plants, Academic Press, London, 1995.

[8] Martin-Prevel P., Rôle des éléments minéraux chez les végétaux, Fruits 33 (1978) 521-529.

[9] Masson P., Influence des différents traitements sylvicoles de la suberaie sur la production et la qualité du liège et sur la protection de la forêt contre les incendies, Rapport de synthèse du contrat européen MA2B CT 910019 (DTEE), 1994.

[10] Oliveira G., Martins-Louçao M.A., Correia O., Catarino F., Nutrient dynamics in crown tissues of cork-oak (Quercus suber L.), Trees 10 (1996) 247-254.

[11] Orgeas J., Bonin G., Variabilité des nutriments foliaires de Quercus suber L. dans différentes situations écologiques dans le massif des Maures (Var, France) et relations avec la production de liège, Ann. Sci. For. 53 (1996) 615-624.

[12] Robert B., Bertoni G., Sayag D., Masson P., Assessment of mineral nutrition of cork-oak through foliar analysis, Commun. Soil. Sci. Plant. Anal. 27 (1996) 2091-2109.

[13] Robert B., Contribution to the study of the cork-oak (Quercus suber L.) mineral nutrition in the natural medium, Thèse Universitat de Girona, ENSAT, 1997.

[14] Zeraia L., Essai d'interprétation comparative des données écologiques, phénologiques et de production suberoligneuse dans les forêts de chêne-liège de Provence Cristalline (France Méridionale) et d'Algérie, Thèse, université sciences et techniques Marseille, 1981. 